\title{
Spatially Developing Secondary Instabilities and Attachment Line Instability in Supersonic Boundary Layers
}

\author{
Fei $\mathrm{Li}^{1}$ \\ National Institute of Aerospace, Hampton, VA 23666 \\ and \\ Meelan M. Choudhari ${ }^{2}$ \\ NASA Langley Research Center, Hampton, VA 23681
}

\begin{abstract}
This paper reports on progress towards developing a spatial stability code for compressible shear flows with two inhomogeneous directions, such as crossflow dominated swept-wing boundary layers and attachment line flows. Certain unique aspects of formulating a spatial, two-dimensional eigenvalue problem for the secondary instability of finite amplitude crossflow vortices are discussed. A primary test case used for parameter study corresponds to the low-speed, NLF-0415(b) airfoil configuration as tested in the ASU Unsteady Wind Tunnel, wherein a spanwise periodic array of roughness elements was placed near the leading edge in order to excite stationary crossflow modes with a specified fundamental wavelength. The two classes of flow conditions selected for this analysis include those for which the roughness array spacing corresponds to either the naturally dominant crossflow wavelength, or a subcritical wavelength that serves to reduce the growth of the naturally excited dominant crossflow modes. Numerical predictions are compared with the measured database, both as indirect validation for the spatial instability analysis and to provide a basis for comparison with a higher Reynolds number, supersonic swept-wing configuration. Application of the eigenvalue analysis to the supersonic configuration reveals that a broad spectrum of stationary crossflow modes can sustain sufficiently strong secondary instabilities as to potentially cause transition over this configuration. Implications of this finding for transition control in swept wing boundary layers are examined. Finally, extension of the spatial stability analysis to supersonic attachment line flows is also considered.
\end{abstract}

Nomenclature

$A_{i} \quad=\quad$ initial amplitude of stationary crossflow vortex

$A, B, C=$ coefficient matrices of the linearized Navier-Stokes equations

$N \quad=\mathrm{N}$ factor

$t \quad=$ time

$N_{y} \quad=$ number of points used in the wall normal direction in the numerical discretization

$N_{\zeta} \quad=$ number of points used in the direction parallel to the wing leading edge in the numerical discretization

$V_{\text {subscripts }}=$ coefficient matrices of the linearized Navier-Stokes equations

\footnotetext{
${ }^{1}$ Present affiliation: Aerospace Engineer, Computational AeroSciences Branch, M.S. 128.

${ }^{2}$ Aerospace Engineer, Computational AeroSciences Branch, M.S. 128, AIAA Associate Fellow.
} 


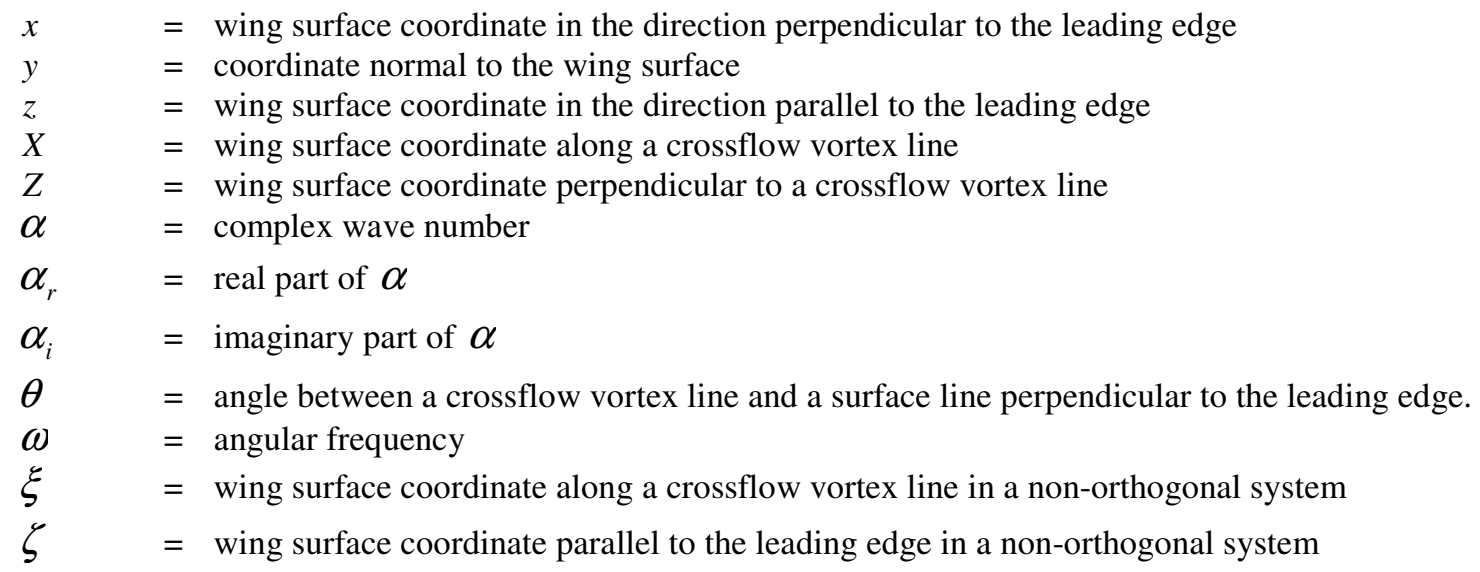

\section{Introduction}

Even in a low-disturbance environment that is characteristic of external aeronautical flows, laminar-turbulent transition in a 3D boundary layer can occur through many routes. Potential paths for transition include the destabilization of attachment line boundary-layer, the development and breakdown of streamwise instabilities (i.e., Tollmien-Schlichting waves and the first and second modes of instability depending on flow Mach number), and the growth and breakdown of crossflow vortex instabilities (if the boundary layer is three-dimensional) or the centrifugal or Goertler mode instability (if the surface geometry includes regions of concave streamwise curvature) $[1,2,3]$. For flow configurations that are dominated by one of the streamwise instabilities, efficient and usually reliable predictions of transition onset can be achieved via linear growth ( $\mathrm{N}$-factor) correlations based on classical stability theory or linear PSE (Parabolized Stability Equations) [4, 5]. However, there is increasing experimental evidence that receptivity and nonlinear processes become relatively more significant in the case of transition due to crossflow or Goertler vortex instabilities [6-9]. A more holistic prediction approach based on the nonlinear evolution of these vortices and the ensuing high-frequency secondary instabilities then becomes desirable [10-12].

An earlier application of a higher-fidelity transition prediction approach [10] had yielded promising results in the context of a low-speed crossflow experiment involving a 45-deg swept NLF-0415(b) airfoil [6]. Specifically, an Nfactor correlation based on the linear amplification of secondary instabilities of stationary crossflow vortices of known amplitude provided a more robust correlation with the measured location for transition onset than an absolute amplitude correlation based on the primary instability alone. The first known application of secondary N-factors in the context of semi-empirical transition prediction was presented by El-Hady in the context of streamwise instabilities in 2D supersonic boundary layers (see, for instance, Ref. [13]). The preliminary results based on this higher fidelity approach were also consistent with the observed delay of transition in the presence of a subcritical stationary crossflow mode that was seeded via artificial, spanwise periodic roughness near the leading edge.

The technique of crossflow-transition control via spanwise periodic roughness is also relevant to supersonic sweptwing flows, but has not yet been demonstrated beyond initial laboratory experiments in the Mach 2.4 Supersonic Wind Tunnel at Arizona State University (ASU) [14]. A preliminary application of the higher fidelity transition prediction approach to the latter experiments was presented in Ref. [11, 12]; however, it did not include an explicit account of the secondary instability phase during transition. A major goal behind the present work is to extend the findings in Ref. [11] by including the growth of high-frequency secondary instabilities of stationary crossflow modes and, more generally, to further develop the holistic prediction approach in the context of compressible boundary layers. To that end, the temporal instability analysis of Refs. [10, 15] is extended to spatial instability modes, allowing systematic mode tracking without any additional simplifying assumptions as required during the temporal analysis. We further note that such spatial predictions can be directly compared with numerical simulations and, perhaps, used to provide suitable initial conditions for a simulation that is focused on the (strongly nonlinear) laminar breakdown phase.

Mathematically, prediction of stationary crossflow transition via the secondary N-factor approach differs from the classical $\mathrm{N}$-factor analysis based on (small-amplitude) primary instability modes, in that the basic state for 
secondary instability analysis (which has been modified by the presence of finite amplitude stationary vortex instabilities) is strongly inhomogeneous in two spatial directions (wall normal and spanwise) instead of just a single direction (wall normal) as during the classical analysis using the unperturbed boundary layer flow as the basic state. Consequently, the amplification characteristics and mode shapes of the high-frequency secondary instability modes are governed by an eigenvalue problem based on a two-dimensional partial differential equation (PDE) [3, 10, 16, 17], rather than the simpler, classical eigenvalue problem that is derived from a system of ordinary differential equations (ODEs). Whereas the latter set of ODEs involves the wall-normal coordinate as the only independent variable, the $2 \mathrm{D}$ eigenvalue problem for the secondary instability of finite amplitude crossflow modes involves an additional, surface-tangential coordinate that cuts across the cross section of the crossflow vortex. In cases where the vortex pattern involves a single dominant wavelength, the secondary perturbations may be treated as being periodic along this latter coordinate. Linear (primary) instabilities of an attachment line boundary layer can also be described using a 2D eigenvalue problem [18-20], except that both the basic state and the unstable perturbations now have an algebraic, rather than periodic, behavior along the second spatial coordinate (along the chordwise direction). Understanding the attachment line instability is of great practical importance in itself; in particular, if the attachment line flow is allowed to become turbulent (due to the growth of instabilities or because of turbulence coming from the fuselage or tripping by roughness), then the rest of the wing surface will become contaminated, rendering it difficult and/or impractical to recover laminar flow.

A number of hydrodynamic instability analyses based on partial differential eigenvalue problems have been reported in the literature (see $[3,10,16-21]$ and the references therein), mostly in the context of the temporal instability (such that the perturbations are spatially periodic and amplify in time) and/or incompressible flows, with only a few analyzed compressible flows (e.g. $[16,19])$. The present paper is focused on the development and application of spatial instability analyses for supersonic swept-wing boundary layers. Both spatially growing primary instabilities along the attachment line and the secondary instabilities of crossflow vortices over an infinite-span airfoil have been considered. Despite their different physical origins, both of the targeted instability mechanisms can be studied using the same analytical and numerical techniques as mentioned in the previous paragraph. Whereas the extension from temporal to spatial predictions is quite trivial for attachment line instability, it is not equally straightforward in the case of the secondary instabilities of crossflow vortices; the additional complexity arises because of the differences in the orientation of the vortex axis (typically within a few degrees of the inviscid streamline) along which the basic state evolves on a slow spatial scale and the direction in which the secondary instability modes amplify (viz., along the chordwise direction). A brief summary of the unique aspects of spatial secondary instability analysis for infinite span swept-airfoil flows is presented in section II below. In section III, we present numerical results pertaining to the low-speed, NLF-0415(b) configuration and compare the predictions with measured data, both as a qualitative validation for the spatial instability predictions and to provide a reference to assess similar results for a supersonic configuration modeling an experiment in the ASU Mach 2.4 Supersonic Wind Tunnel (Section IV) [14]. Additional benchmarking and application of the spatial instability code in the context of supersonic attachment line boundary layers is outlined in section V. Concluding remarks are presented in section VI.

\section{Formulation of Secondary Instability Equations and Solution Precedure.}

The equations governing the unstable perturbations are obtained by linearizing the Navier-Stokes equations about a specified basic state, e.g., a finite amplitude crossflow vortex developing in a swept-airfoil boundary layer. For a basic state that is slowly varying along one spatial coordinate (viz., the vortex axis in the case of crossflow vortices), using a wave ansatz in that direction for the disturbance quantities (in the spirit of WKB or multiple scale analysis) reduces the spatial dimension of the problem by one, resulting in a set of two-dimensional, linear partial differential equations at the leading order. In the case of temporal secondary instability analysis for stationary crossflow vortices in a swept airfoil boundary layer $[3,10,16]$, the coordinate system is usually rotated so that one of the coordinate axes along the surface is aligned with the local vortex direction $(X)$ and the other surface coordinate

$(Z)$ is orthogonal to $X$ (Fig. 2.1). This leads to a set of linear partial differential equations in terms of the surface normal coordinate $Y$ and the across-the-vortex coordinate $Z$. Together with appropriate boundary conditions at the wall and free-stream boundaries, and periodic boundary conditions in $Z$, one is led to a planar (i.e., 2D) eigenvalue problem which may be solved using numerical techniques for large linear systems. 
The above choice of surface coordinates becomes inappropriate in the context of spatial instability analysis. Specifically, the spatial exponential growth obtained from the 2-D eigenvalue problem in the coordinate system $(X, Z)$ is in the direction of vortex axis and, therefore, has a component in the direction parallel to the leading edge, which is not the solution we desire. In addition, the base flow is not periodic in $Z$ so that approximations have to be made based on the fact that the deviation from periodicity is small. What we look for is a solution that is periodic in the direction parallel to the leading edge ( $\zeta$ or $z$-direction in Figure 2.1) and grows only in the direction perpendicular to the leading edge ( $x$-direction in Figure 2.1). For infinite-span configurations, the unperturbed boundary layer is invariant in the direction $z$ parallel to the leading edge (Fig. 2.1), which allows both the primary (i.e., the stationary crossflow vortex) and the secondary disturbance fields to be periodic in that direction. However, because the primary disturbance field exhibits "rapid" variation in the chordwise coordinate $x$, the orthogonal coordinate system $(x, z)$ cannot be used to reduce the spatial secondary instability analysis to a $2 \mathrm{D}$ eigenvalue problem. However, the non-orthogonal, vortex aligned coordinate system $\xi$ and $\zeta$ (where $\xi=X$ and $\zeta=z$ ) can be used to formulate the spatial secondary instability problem as sketched below. We note that the non-orthogonal formulation precisely mimics the DNS computations in refs. [22-23], wherein the vortex aligned non-orthogonal coordinate system was employed towards efficient simulation of disturbance evolution in swept airfoil boundary layers without any approximations. For general 3D configurations, the implementaton of parabolized stability equations (PSE) in the LASTRAC code [24] also allows the option of a non-orthogonal coordinate system.

The linearized compressible Navier-Stokes equations in the non-orthogonal system can be written in the following form

$$
\begin{aligned}
& \Gamma \frac{\partial \phi}{\partial t}+A \frac{\partial \phi}{\partial \xi}+B \frac{\partial \phi}{\partial y}+C \frac{\partial \phi}{\partial \zeta} \\
& =V_{\xi \xi} \frac{\partial^{2} \phi}{\partial \xi^{2}}+V_{y y} \frac{\partial^{2} \phi}{\partial y^{2}}+V_{\zeta \zeta} \frac{\partial^{2} \phi}{\partial \zeta^{2}}+V_{\xi y} \frac{\partial^{2} \phi}{\partial \xi \partial y}+V_{y \zeta} \frac{\partial^{2} \phi}{\partial y \partial \zeta}+V_{\xi \zeta} \frac{\partial^{2} \phi}{\partial \xi \partial \zeta}
\end{aligned}
$$

where $\phi$ is a vector whose elements are the perturbation pressure, velocities and temperature, the coefficients of the derivatives are $5 \times 5$ matrices that are slow varying functions of $\xi$, functions of $y$ and periodic functions of $\zeta$.

In the surface coordinate system $(\xi, \zeta)$, the base flow is slowly varying in the $\xi$-direction and periodic in the $\zeta$ direction. The perturbation variables associated with the secondary instability can then be expressed in the form

$$
\phi(\xi, y, \zeta)=\varphi(\xi, y, \zeta) \exp \left(-i \omega t+i \int \alpha d \xi\right)
$$

where $y$ is the coordinate normal to the wing surface, $\varphi(\xi, y, \zeta)$ is the 2-D complex eigenfunction at station $\xi$ that is periodic in $\zeta ; \omega$ is the given frequency; and $\alpha$ is the complex wave number (i.e., the spatial eigenvalue). We emphasize that, in the non-orthogonal coordinate system, the use of $\alpha d \xi$ in the exponential part does not constitute allowing the disturbance to grow in the spanwise $(z)$ direction. This point will become clearer later on when we write the expression for the evolution of perturbation quantities in the more intuitive orthogonal coordinate system.

On substituting Eq. (2) into Eq. (1) and neglecting the dependencies on $\xi$ of both the coefficients of the equations and the mode shape $\varphi$, we obtain 


$$
\begin{aligned}
& -i \omega \Gamma \varphi+\left(i \alpha A+\alpha^{2} V_{\xi \xi}\right) \varphi+\left(B-i \alpha V_{\xi y}\right) \frac{\partial \varphi}{\partial y}+\left(C-i \alpha V_{\xi \zeta}\right) \frac{\partial \varphi}{\partial \zeta} \\
& =V_{y y} \frac{\partial^{2} \varphi}{\partial y^{2}}+V_{\zeta \zeta} \frac{\partial^{2} \varphi}{\partial \zeta^{2}}+V_{y \zeta} \frac{\partial^{2} \varphi}{\partial y \partial \zeta}
\end{aligned}
$$

Equation (3) is solved as a 2-D spatial eigenvalue problem with $\alpha$ as the eigenvalue for a given $\omega$. Periodic boundary condition is used in the $\zeta$ direction and vanishing velocity and temperature conditions are used at the wall, along with suitable boundary conditions along the free-stream boundary.

The form of the solution in Eq. (2) is not intuitive and, for better clarity, we can rewrite it in terms of the more intuitive orthogonal coordinates. To this end, it is easy to verify that the surface coordinate systems $(x, z)$ and $(\xi, \zeta)$ are related through the following expressions

$$
\begin{aligned}
& d \xi=\frac{d x}{\cos \theta} \\
& d \zeta=d z-d x \tan \theta
\end{aligned}
$$

where $\theta$ denotes the angle between the $\xi$ and the $x$ axes. Therefore, the right hand side of Eq. (2) can be written as $\varphi\left(\int \frac{d x}{\cos \theta}, y, z-\int \tan \theta d x\right) \exp \left(-i \omega t+i \int \frac{\alpha_{r} d x}{\cos \theta}-\int \frac{\alpha_{i} d x}{\cos \theta}\right)$ where $\alpha_{r}$ and $\alpha_{i}$ are, respectively, the real and imaginary parts of $\alpha$. Observe that, at any fixed $x, \varphi$ is a periodic function of $z$; the solution oscillates in $x$ along with an exponential growth or decay in its amplitude; and, furthermore, there is no exponential growth in $z$. The $\mathrm{N}$-factor is then defined as

$$
N=-\int \frac{\alpha_{i} d x}{\cos \theta}
$$

where the lower limit of integration corresponds to the onset of the instability.

The formulation is also valid when the base flow, and hence the coefficient matices, are not periodic in $\zeta$, in which case the eigenfunction $\varphi(\xi, y, \zeta)$ is not periodic and an alterative set of boundary conditions need to be applied.

Numerically, Equation (3) is discretized in the $y$-direction using high order finite difference and, when the coefficient matrices are periodic, discrete Fourier spectral method is used in the $\zeta$-direction, otherwise high order finite difference is used. The resulting algebraic eigenvalue problem has a dimension of $5 \times N_{\zeta} \times N_{y}$, however, its banded structure can be utilized for storage and computational efficiency. The algorithms used for solving the algebraic eigenvalue prolem are the same as those used in previous analyses of $2 \mathrm{D}$ eigenvalue problems (e.g. [10]).

A typical $\mathrm{N}$-factor computation procedure involves several steps:

(0) Computation of basic state for secondary instability: The unperturbed boundary-layer flow is computed by solving compressible boundary-layer equations for a specified inviscid pressure distribution that is derived from Euler calculations. The evolution of stationary crossflow vortices in this flow is computed using nonlinear Parabolized Stability Equations (PSE) as implemented in the Langley Stability and Transition Analysis Code (LASTRAC) [24]. The wall-normal resolution used for these computations varied between 121 to 251 points, and between 64 to 96 Fourier modes were typically used in the spanwise direction. For secondary instability calculations, the basic state computed with nonlinear PSE was interpolated onto a grid that is more suitable for secondary instability modes. A typical resolution used for the eigenvalue 
computations presented in this paper corresponds to 121 points in the wall-normal direction and 32 points in the periodic $(\zeta$ ) direction, although additional resolution was found to be necessary in some of the cases. The computational grid was tailored to the anticipated/computed mode shapes and spot checks were employed to verify that the shift in eigenvalues was insignificant when the number of points was further increased and/or when the clustering associated with the wall-normal grid was modified.

(1) A thorough eigenvalue search is performed at one or more chordwise locations for a range of wavenumbers. The Arnoldi method is used for global computations of temporal eigenvalues which, in turn, are used as starting values for an iterative spatial computation. This establishes the number of unstable modes and the corresponding range of frequencies.

(2) A suitable subset of eigenvalues for each mode is chosen to span the relevant range of frequencies. This forms the starting set of eigenvalues to be used for $\mathrm{N}$-factor calculations for that mode.

(3) With each selected eigenvalue from step (2) as a starting guess, the eigenvalue computation is marched both upstream and downstream in the chordwise direction to cover the appropriate range of locations. At each step during the marching process, the starting guess is updated via linear extrapolation from the previously computed eigenvalues at adjacent locations. Having determined the eigenvalues over the region of interest, the $\mathrm{N}$-factor evolution for each frequency and mode type is calculated using Eq. (6).

\section{Secondary Instability of Crossflow Vortices: Low Mach Number NLF-0415b Case}

We first examine the secondary instability of crossflow vortices for the NLF-0415b configuration that was used for detailed measurements of crossflow induced transition in the Arizona State University (ASU) Unsteady Wind Tunnel [25]. During those experiments, a spanwise periodic array of roughness elements was placed near the airfoil leading edge to introduce stationary crossflow vortices with the desired wavelength and varying initial amplitude. Herein, we choose to model two sets of experimental data points, corresponding to vortex wavelengths of $12 \mathrm{~mm}$ (the naturally dominant stationary crossflow mode) and $8 \mathrm{~mm}$ (subdominant mode that was found to weaken the growth of the naturally excited $12 \mathrm{~mm}$ mode and, hence, to delay the onset of transition on the airfoil), respectively, at the fixed chord Reynolds number of 2.4 million. Similar to [10], initial amplitudes of the stationary vortex are chosen so that the computational amplitudes at a selected upstream location are closely matched with the experimental results for three roughness heights of 6,18 and 48 microns, respectively, for the $12 \mathrm{~mm}$ case. Although the roughness induced receptivity is not directly modeled in this paper, for convenience of notation, we will refer to these cases based on the corresponding experimental configuration. Accordingly, the above three cases are denoted as H06S12, H18S12 and H48S12, respectively. Similarly, for the $8 \mathrm{~mm}$ case, two roughness heights of 6 and 48 microns are chosen to match the computational results. These are referred to as H06S08 and H48S08, respectively. We note that the stabilizing effect of a higher amplitude $8 \mathrm{~mm}$ mode on the $12 \mathrm{~mm}$ stationary mode has already been established in both experiments [27] and computations [10]. Thus, the goal behind the $\lambda_{\mathrm{z}}=8 \mathrm{~mm}$ computations presented in this paper is to examine the growth potential for secondary instabilities at that wavelength and their likely effect on the transition onset location.

In Fig. 3.1a, predicted modal amplitudes for the leading three harmonics are compared with hot wire measurements for the H18S12 case, where 18 micron roughness elements were applied at $\lambda_{\mathrm{z}}=12 \mathrm{~mm}$. Effect of varying roughness height on the fundamental disturbance amplitudes is depicted in Fig. 3.1b. An analogous comparison for the $12 \mathrm{~mm}$ cases was presented in [10], wherein the secondary instability of the nonlinear stationary crossflow vortices was examined using temporal analysis. Because an envelope method was used to compute the integrated amplification of the secondary instabilities in [10], (unlike the present spatial analysis) the computed $\mathrm{N}$-factors did not track the growth of a fixed disturbance entity. We also note that, whereas the unperturbed boundary layer flow in [10] had been predicted using the measured surface pressure distribution, the present set of results is based on a $\mathrm{Cp}$ distribution obtained by solving Euler equations for the conditions of interest.

$\mathrm{N}$-factor evolution for the unstable modes at various selected frequencies are plotted in Figure 3.2 for each of the selected $12 \mathrm{~mm}$ cases. Corresponding growth rates for the 18-micron roughness (H18S12) case are also shown for illustration. Several secondary instability modes are found to exist in each of these cases, of which a few relevant modes were selected for plotting in Figure 3.2 and are represented by green, pink, and blue colors, respectively. The red curves denote lower frequency modes that originate significantly farther upstream as the traveling crossflow modes of the unperturbed boundary-layer flow but are substantially modified in the downstream region due to the finite amplitude stationary crossflow instability. 
The first set of (dominant) high-frequency secondary instability modes to become unstable is denoted by the green curves. It was determined from the relative contributions to the energy production terms that the green modes are associated with the wall-normal shear of the basic state (i.e. represent a $y$-mode in the notation of [10]) and attain a peak growth rate at around $\mathrm{f}=4.7 \mathrm{kHz}$ for the 6 - and 18 -micron roughness cases and around $\mathrm{f}=4.9 \mathrm{kHz}$ for the case of highest initial crossflow amplitude (i.e., 48 micron roughness case). This mode is denoted as y-mode. An additional set of modes (denoted via pink curves) becomes unstable either at about the same location as the y-mode, or somewhat farther downstream, depending on the roughness height used. This family of modes is associated with the spanwise shear term and, hence, is denoted as $z$-mode 1 in this paper. It has a peak frequency of $1.6 \mathrm{kHz}$ for lower initial amplitudes of the crossflow vortex and $2.9 \mathrm{kHz}$ for the case with the highest initial crossflow amplitude. Subsequently, however, a second set of z-modes (denoted via blue curves) becomes unstable and achieves much higher growth rates (and, hence, $\mathrm{N}$-factors) in comparison with the first $\mathrm{z}$-mode. The peak growth rate of this dominant set of $\mathrm{z}$-modes ( $\mathrm{z}$-mode 2 ) is found to be near $\mathrm{f}=3.4 \mathrm{kHz}$ for nearly all initial crossflow vortex amplitudes.

We observe that the maximum growth rate of the y-mode (denoted by the green curve) occurs between $x / c=0.3$ and 0.4 , while the peak growth of the dominant $\mathrm{z}$-mode (denoted by blue curve) occurs in bewteen $x / c=0.45$ and 0.5 . At the measured transition location of $x / c=0.52$ for the 6-micron roughness case, these $\mathrm{y}$ - and z-modes reach $\mathrm{N}$ factors of approximately 10 and 7, respectively. As the roughness height (or, equivalently, the initial crossflow amplitude) is increased, each of these secondary instability modes become unstable at progressively upstream locations; however, the corresponding peak growth rates are found to decrease at the same time. The net result is that the transition $\mathrm{N}$-factor attained by the $\mathrm{y}$-mode 1 is increased to approximately 12 , while that reached by the $\mathrm{z}$ mode 2 is reduced to approximately 6 for the highest initial crossflow amplitude. $\mathrm{N}$-factors for the intermediate roughness height (case H18S12) fall in between the corresponding values for the H6S12 and H48S12 cases, so that the overall spread in transition $\mathrm{N}$-factors for either of the above two modes is small. The present $\mathrm{N}$ factors for the $\mathrm{z}$ mode are somewhat lower in comparison with [10]; however, the precise cause behind this difference could not be ascertained.

The above findings confirm the strong correlation between the measured transition location and the predicted secondary $\mathrm{N}$-factor, regardless of whether the $\mathrm{N}$-factor correlation is based on the amplification of the $\mathrm{y}$-modes (which were found to be insignificant to transition in the related measurements by White and Saric [28]) or the zmodes of secondary instability (which were found to be an important catalyst for initiating the process of laminar breakdown in [28]). Detailed measurements in [28] showed the peak fluctuations associated with the z-mode to be slightly above $3 \mathrm{kHz}$, which is consistent with the most amplified z-type secondary instability modes as predicted in the current analysis. The corresponding mode shapes for the dominant $z$-modes were also found to be rather close (compare, for instance, the mode shape of z-mode 2 at the bottom of Fig. 3.5 with Fig. 10 from ref. [28]). The reason(s) why the y-modes of secondary instability did not play an active role during transition in the experiment may be related to the receptivity characteristics pertaining to this mode; however, additional work is necessary to clarify those reasons. The recent work by Bonfigli and Klocker [26] appears to offer partial clues in this regard.

The measurements in [28] indicated an additional spectral peak near $200 \mathrm{~Hz}$ that corresponds to traveling crossflow vortices modulated by the spanwise variations associated with stationary crossflow mode $[3,26]$. At the larger roughness height (i.e., larger initial stationary crossflow amplitude), the low frequency traveling disturbances attained significantly large amplitudes. Just prior to the laminar breakdown, the amplitudes of the low-frequency traveling modes were comparable to those of the z-modes of seconday instability, so it's possible that both types of modes jointly contributed to the onset of breakdown at the above test condition. The behavior of the low-frequency traveling modes for $\lambda_{z}=12 \mathrm{~mm}$ (cases H6S12 through H48S12) is investigated next.

We note that the low-frequency modes are unstable even in the absence of the stationary crossflow vortices. However, the stationary crossflow vortices, after they attain a finite amplitude, can significantly modify the behavior of these traveling modes. The red curves in Figure 3.2 show the $\mathrm{N}$-factors and the growth rates of the traveling waves. Direct comparisons of the $\mathrm{N}$-factors and growth rates of the modulated traveling waves for all three roughness heights are shown in Figure 3.3. These traveling waves start to become unstable approximately at $x / c=0.04$, and begin to show the effects of roughness height at approximately $x / c=0.07$, obviously because the presence of the growing stationary vortices is beginning to be felt at that stage. Up to $x / c=0.2$, the maximum $\mathrm{N}$ factor for the traveling waves is lowest in the case of stationary crossflow vortices with the highest initial amplitude 
(blue set of curves in Fig. 3.3a). Based on the energy production budget, we find that the traveling waves in the upstream region $(x / c<0.15)$ are primarily associated with the wall-normal shear of the basic state (i.e., these modes correspond to $y$-modes). This is to be expected because they originate from a region in which the flow field is nearly uniform in the z-direction.

The upstream peak in the growth rate curves (near $x / c=0.10 \rightarrow 0.12$ ) corresponds to frequencies in the vicinity of $150 \mathrm{~Hz}$. The initially dominant set of traveling waves have lower amplification rates in the region downstream of the of first lobe in the growth rate curves. Traveling modes near $460 \mathrm{~Hz}$ attain their peak growth rates between $x / c=$ 0.25 and $x / c=0,36$ depending on the initial amplitude of the stationary crossflow mode (i.e., the roughness height parameter). The second lobe in the growth rate curve from Fig. 3.3b is associated with the spanwise shear of the basic state (which is induced by the relatively strong stationary crossflow vortex). Thus, it is not surprising that the peak of this second lobe shifts progressively upstream as the roughness height is increased from 6 micron (red curves in Fig. 3.3b) to 48 micron (blue curves in Fig. 3.3b).

To illustrate the differences between the structures of traveling crossflow vortices in the upstream and downsteam regions, respectively, we next examine the mode shape evolution for a traveling mode at $\mathrm{f}=200 \mathrm{~Hz}$ in the $\mathrm{H} 06 \mathrm{~S} 12$ case (Fig. 3.4). This particular traveling wave is seen to be a $y$-mode at small $x / c$ and transitions to a $z$-mode beyond $x / c=0.25$. The amplitudes of these waves are concentrated closer to the wall than the secondary instability waves, which ride on parts of the crosssflow vortices that are farther away from the wall as shown in Figure 3.5 for the 18 micron case. The mode shape of the modulated traveling wave looks quite similar to the spatial distribution of low frequency oscillations observed in the experiment of White and Saric [28] (compare, for example, the mode shape shown at the bottom of Fig. 3.4 here with Fig. 7 of reference [28]).

Because the computational resources required for secondary instability analysis can exceed the resources required for primary instability analysis by an order of magnitude or greater, it is worthwhile to examine if the onset of secondary instability may be correlated with certain features of the primary flowfield. As a partial attempt towards that goal, we now examine the spanwise averaged velocity profiles (obtained by using nonlinear PSE analysis) along the direction of the inviscid streamline at a few select streamwise locations for each of the three cases examined above (Fig. 3.6). As may be expected, the onset of the y-mode (high-frequency) secondary instability approximately coincides with the locations where the spanwise averaged velocity profile (aligned with the inviscid streamline at the boundar layer edge) first develops a visually distinct inflection point higher up in the boundary layer. For ease of comparison, those profiles (corresponding to $x / c \approx 0.35,0.30$ and 0.25 for the 6,18 and 48 micron cases, respectively) have been highlighted in Fig. 3.6.

Next, we consider the effect of $8 \mathrm{~mm}$ stationary crossflow waves on both traveling waves with the same spanwise wavelength and the high frequency secondary instability. Again, the initial amplitudes of the $\lambda_{\mathrm{z}}=8 \mathrm{~mm}$ mode have been chosen to achieve an approximate match between the overall predicted evolution of the r.m.s. primary amplitudes and the corresponding measured data for roughness heights of 6 micron and 48 micron, respectively (Fig. 3.7). Carrillo et al. [27] report that the controlled excitation of stationary crossflow modes at a subcritical spacing delayed the onset of transition to $x / c=0.81$ at the lower roughness height $(\mathrm{h}=6$ microns); however, the transition front moved back upstream to $\mathrm{x} / \mathrm{c}=0.61$ as the roughness height increased to $\mathrm{h}=48 \mathrm{microns}$ (case $\mathrm{H} 48 \mathrm{~S} 8$ ). We try to gain some insight into these findings by examining the behavior of secondary instabilities in each of these cases.

Figures 3.8a and 3.8b show the $\mathrm{N}$-factors of the non-stationary modes with $\lambda_{\mathrm{z}}=8 \mathrm{~mm}$ for the above two cases. For $\mathrm{h}=6$ microns (case H6S8), the traveling primary waves dominate, reaching an N-factor of approximately 6 at $\mathrm{x} / \mathrm{c}=0.6$, while the two secondary instability modes (a y-mode and a $\mathrm{z}$-mode) barely show up during the region of significant primary amplitudes at $\lambda_{\mathrm{z}}=8 \mathrm{~mm}$. At the larger initial crossflow amplitude corresponding to $\mathrm{h}=48$ microns, the growth of the traveling crossflow modes is significantly reduced $(\mathrm{N} \approx 4$ near $x / c=0.6)$; but, the $\mathrm{y}-$ and $\mathrm{z}-$ modes of secondary instability reach significantly larger maximum $\mathrm{N}$ factors $(\mathrm{N}=6$ and $\mathrm{N}=2.5$, respectively) relative to the $\mathrm{h}=6$ micron case. These computational findings suggest that there is little danger of premature transition due to the 8-mm control input when $\mathrm{h}=6$ microns (consistent with the large transition delay measured in the experiment), but there exists the possibility of an adverse impact on the transition location due to the relatively stronger secondary instability in the $\mathrm{h}=48$ micron case (especially if the $\mathrm{y}$-modes can get naturally excited at the smaller vortex wavelength). Whether or not this adverse effect may have been responsible for the upstream shift in the measured 
transition location (from $\mathrm{x} / \mathrm{c}=0.81$ for $\mathrm{H} 6 \mathrm{~S} 8$ case to $\mathrm{x} / \mathrm{c}=0.61$ for H48S8) cannot, however, be established on the basis of the present analysis alone.

\section{Spatial Secondary Instabilities of Compressible Stationary Crossflow Vortices}

Next, we present selected results for a Mach 2.4, 73-degree swept wing geometry that was modeled after the experimental configuration of Saric and Reed [14]. The nonlinear development of stationary crossflow vortices with various spanwise wavelengths and initial amplitudes were computed in Ref. [11] for a chord Reynolds number of $\mathrm{Re}_{\mathrm{c}}=16$ million. The dominant stationary crossflow mode in this case corresponds to a spanwise wavelength of 3 $\mathrm{mm}$. The first harmonic of this naturally dominant mode $(1.5 \mathrm{~mm}$ spanwise wavelength) provides the simplest (although not optimal) form of control input to weaken the growth of the naturally dominant stationary modes. Determination of appropriate control input parameters (e.g., wavelength and amplitude of the control mode) is, of course, a major issue in designing an effective laminar flow control system. Specifically, the control input must be large enough to provide the desired control action (viz., sufficient modification of the basis state in order to induce the required stabilization of the "dangerous" modes), however, it cannot be excessively large as to precipitate premature transition (and/or reduce the extent of transition delay, as briefly alluded to in section III in the context of the experiments in [27]). As shown below, the secondary instability analysis (in conjunction with receptivity predictions [29]) may provide useful guidance to help select an appropriate range of control input amplitudes.

The streamwise evolution of primary disturbance and mean flow distortion of the stationary mode with $\lambda_{\mathrm{z}}=3 \mathrm{~mm}$ with an initial amplitude of $A_{i}=10^{-7}$ is plotted in Fig. 4.1a, and the analogous evolution of the $\lambda_{z}=1.5 \mathrm{~mm}$ mode with several initial amplitudes is shown in Fig. 4.1(b). As discussed in Ref. [11], $\lambda_{z}=3 \mathrm{~mm}$, the amplitude of the dominant mode rises quite rapidly along the chordwise direction, even when the amplitude is large enough to induce high-frequency secondary instability. The amplitude of the subcritical mode (Fig. 4.1b) reaches its peak significantly earlier than the $\lambda_{z}=3 \mathrm{~mm}$ mode (Fig. 4.1a) and decreases rapidly thereafter. Thus, both the control action and the potential for premature transition due to such subcritical modes is limited to a finite spatial region in the vicinity of the modal peak.

Amplification characteristics of secondary instability modes for the $\lambda_{z}=3 \mathrm{~mm}$ case are shown in Fig. 4.2. The Nfactor curves for a range of selected frequencies are plotted in Fig. 4.2(a), whereas Fig. 4.2(b) shows the corresponding spatial growth rates. High-frequency secondary instability modes are seen to exist for frequencies up to $2000 \mathrm{kHz}$. The maximum $\mathrm{N}$-factor for the secondary modes reaches 10 even before the peak primary amplitude has been reached, indicating that (if, indeed, the primary instability spectrum were to mirror the inflow behavior in Fig. 4.2(a) and the disturbance environment can excite the relevant secondary instability modes) the onset of transition should occur near $\mathrm{x} / \mathrm{c} \approx 0.6$. The growth characteristics of the secondary modes also support the hypothesis [11] that, in this particular case, movements in transition onset might approximately correlate with the corresponding shift in the region of rapid rise in the primary amplitude.

A more detailed examination of the $\lambda_{z}=3 \mathrm{~mm}$ case reveals that there exist at least four secondary instability modes, all of which are y-modes and two are shown in Fig. 4.2 (b). A y-mode is the first to become unstable at approximately $x / c=0.5$, this mode reaches an $\mathrm{N}$-factor of approximately 8.5 for a frequency of approximately 1.05 $\mathrm{MHz}$ at $x / c=0.60$. However, transition is likely to be caused by a second y-mode with a frequency near $1.3 \mathrm{MHz}$, which becomes unstable at $x / c \approx 0.55$, overtakes the y-mode 1 in terms of $\mathrm{N}$-factor near $x / c=0.57$ and reaches a maximum $\mathrm{N}$-factor of approximately 10 at $x / c=0.60$. The other two y-modes (not shown) reach much lower $\mathrm{N}$ factors.

We next examine the variation in secondary instability $\mathrm{N}$-factors as the initial amplitude of the control mode $\left(\lambda_{z}=\right.$ $1.5 \mathrm{~mm}$ ) is varied (assuming this mode alone to be dominant over the relevant spatial region). The effect of modulation of primary instability by stationary crossflow vortices of initial amplitudes of $0.001,0.002$ and 0.005 is shown in Figure 4.3, and secondary instability results for initial amplitudes of $A_{i}=0.001$ and 0.002 (which yields a modest stabilization of the $\lambda_{z}=3 \mathrm{~mm}$ stationary mode as described in [11]) are shown in Figs. 4.4(a)-(b) and 4.4(c)(d), respectively. 
Figure 4.3 shows that the growth rates of the modulated primary instability immersed in higher amplitude crossflow vortices have lower growth rates up to $x / c \approx 0.14$, indicating the weak stabilizing influence of moderate amplitude stationary crossflow mode on lower amplitude traveling crossflow modes. Farther downstream, however, this trend is reversed. Higher amplitude stationary crossflow vortices now appear to enhance the linear growth of the traveling modes that are dominant in the downstream region, as reflected by the progressively upstream shift in the (local) peak in the growth rate curves as the roughness height is increased (similar to the low-speed case examined in section III). However, the linear growth mechanism takes on different characteristics as the growing traveling modes can now be either z-modes and/or y-modes.

For $\lambda_{z}=1.5 \mathrm{~mm}$, up to six different high-frequency secondary instability modes were found, with three predominant ones (Figure 4.4). Two of those three modes are y-modes and the remaining one is a $\mathrm{z}$-mode. They are shown via different colors in Figure 4.4. For the $A_{i}=0.001$ case, the three modes have peak frequencies of $0.88,1.72$ and 0.72 $\mathrm{MHz}$, respectively, and for the $\mathrm{A}_{\mathrm{i}}=0.002$ case, those frequencies increase to $1.16,2.0$ and $1.56 \mathrm{MHz}$, respectively. For an initial amplitude of 0.001 , the maximum $\mathrm{N}$-factor approaches 9 for one of the y-modes. For an initial amplitude of 0.002 , the $\mathrm{N}$-factor reaches approximately 13. All of these findings indicate a strong possibility of premature transition at this level of control input. The finding that strong secondary instabilities can occur at both $\lambda_{\mathrm{z}}=3 \mathrm{~mm}$ and $\lambda_{\mathrm{z}}=1.5 \mathrm{~mm}$ (and, therefore, at the intermediate wavelengths as well) is suggestive of the potentially delicate nature of roughness based transition control at higher chord Reynolds numbers.

The progressive distortion of the mode shape corresponding to the (primary) traveling crossflow modes over this supersonic configuration is indicated in Fig. 4.5. The distorted mode shape of the traveling crossflow mode at $x / c=0.25$ may be compared with the velocity contours of the base flow (top of Fig. 4.6) and the local mode shapes of the dominant secondary instability modes (upper and lower middle, and bottom of Fig.4.6). The associated energy production budget reveals that both the wall-normal and spanwise shears of the basic state make comparable contributions to the growth rate of this traveling crossflow mode at $x / c=0.25$.

Similar to the NLF-0415(b) case, the onset of the first y-associated secondary instability correlates well with the first appearance of inflections in the mean momentum profiles near the boundary layer edge. For the $3 \mathrm{~mm}$ case, the inflection first appears at approximately $x / c=0.55$, which is also where the mode that reaches the highest $\mathrm{N}$-factor becomes unstable. Even though another y-mode becomes unstable prior to $x / c=0.55$, it does not attain a significantly large $\mathrm{N}$-factor until much later in comparison with the dominant mode of secondary instability (Fig 4.2). For the $1.5 \mathrm{~mm}$ cases, the inflections occur at $x / c=0.22$ and 0.18 , respectively, for the two initial amplitudes considered herein (Fig. 4.7). Again, the onset of inflection approximately coincides with the onset of the y-type secondary instability.

\section{Attachment Line Instabilities in a Supersonic Boundary Layer}

As previously noted, instabilities of an attachment line flow are also governed by a $2 \mathrm{D}$ eigenvalue problem that resembles the formulation outlined in section II for the secondary instabilities of crossflow vortices. Therefore, the incompressible swept Hiemenz flow configuration of Lin and Malik [18] was used as an additional test case to benchmark the accuracy of the two-dimensional eigenvalue solver. Having verified the agreement between our predictions and their temporal results (to at least 5 significant digits), spatial eigenvalues for the same case were computed. The spatial equivalent of the $S 1, A 1, S 1$ and $A 2$ modes in Lin and Malik [18] are shown in Figure 5.1, where $S$ and $A$ indicate the symmetric or antisymmetric nature of the mode shape with respect to the attachment line, and the numerical suffix denotes the modal index within each family.

Analogous results for a supersonic attachment line boundary layer with a boundary-layer edge Mach number of $\mathrm{M}=1.69$ are plotted in Fig. 5.2(a) for different values of the attachment line Reynolds number $(\bar{R})$. The mean flow profiles are based on an approximate solution to the Navier-Stokes equations [30] that should be valid sufficiently close to the attachment line. The selected Mach number is expected to be similar to the experimental conditions of Powell et al [31], who studied oversuction effects associated with discrete wall suction across a perforated skin on attachment line transition. The main goal of their experiment was to investigate how the orientation of suction hole rows with respect to the attachment line influenced transition at suction levels relevant to supersonic flight configurations. Present computations do not address the effects wall suction (either in discrete or continuous form) 
on the attachment line flow; however, they provide some guidance with respect to the instability characteristics for the baseline (i.e., zero-suction) case.

The growth rates of the 2D instability modes decrease as the Mach number is increased, so that only the leading symmetric mode (mode $\mathrm{S} 1$ ) is unstable up to $\bar{R}=1000$ in the $\mathrm{M}=1.69$ case. The critical Reynolds number below which the attachment line flow becomes stable to $2 \mathrm{D}$ instability modes is between 600 and 650 . Eigenfunctions at

the attachment line location for the $\bar{R}=1000$ case are plotted in Figure 5.2(b). One observes that the temperature fluctuations have emerged as the dominant fluctuations, displaying a slightly higher peak than the velocity fluctuations along the attachment line. To draw more definitive conclusions regarding the overall instability of the flow, however, we will need to extend the above computations to 3D instability modes which are likely to be more unstable than the 2D modes at this Mach number.

\section{Conclusions}

We have developed planar (2D) eigenvalue analysis capability for spatial instabilities of compressible shear flows with two inhomogeneous directions, such as crossflow dominated swept-wing boundary layers and attachment line flows. Direct spatial 2-D eigenvalue computations, rather than approximate calculations based on a combination of temporal analysis and the well-known Gaster's relation, were performed to characterize the spatial growth of secondary instabilities of stationary crossflow vortices. The equations governing these instabilities were formulated in a non-orthogonal coordinate system, so that the proper periodic boundary conditions may be imposed in the direction parallel to the leading edge of an infinite-span swept airfoil and the exponential growth in the direction perpendicular to the leading edge can be computed without any ambiguity.

The choice of numerical case studies was motivated by transition prediction and control for swept wing boundary layers. The selected swept-airfoil configurations included (i) the low-speed, 45-degree sweep, NLF-0415(b) configuration with a chord Reynolds of $\operatorname{Re}_{\mathrm{c}}=2.4$ million, as tested previously in the ASU Unsteady Wind Tunnel, and (ii) a Mach 2.4, 73-degree sweep configuration with $\mathrm{Re}_{\mathrm{c}}=16$ million. For each of these configurations, we examined both the modification of the traveling crossflow modes and the destabilization of high-frequency secondary instabilities in the presence of finite amplitude stationary crossflow vortices of a specified spanwise wavelength and varying initial amplitudes.

Regardless of the speed and/or the chord Reynolds number, the onset of secondary instability was found to move forward as the initial amplitude of the stationary crossflow vortex was increased. As the stationary vortex increased in amplitude along the downstream direction, the base flow momentum profiles along the direction of the inviscid streamline was shown to develop a visually inflectional character higher up in the boundary layer and the locations for the onset of the high-frequency secondary instability seemed to correlate with the emergence of this inflection point.

The stationary crossflow vortices also modulate any small-amplitude traveling crossflow vortices, resulting in a weak stabilization of the initially dominant traveling modes. The initial stabilizing effect is more pronounced for higher initial amplitudes of the stationary crossflow vortex. Farther downstream, however, the above trend is reversed such that the most unstable traveling modes in that region are actually destabilized as a result of the stationary mode.

Overall, the results reconfirm that secondary instability analysis can explain a number of observed features during the previous experiments in the ASU Unsteady Wind Tunnel, both with and without the use of surface roughness at a subdominant wavelength as a means of transition control. Application of the $2 \mathrm{D}$ eigenvalue analysis to the higher Reynolds number, supersonic configuration revealed that a broad spectrum of stationary crossflow modes (including modes that would be suitable for roughness based transition control) can sustain sufficiently strong secondary instabilities, so as to potentially induce transition over this configuration. Implications of this finding for transition control in swept wing boundary layers were examined. Finally, extension of the spatial stability analysis to supersonic attachment line flows was also considered. Future work should include the incorporation of nonparallel effects into the eigenvalue problem formulations. Additionally, PSE and DNS computations would be helpful to clarify and confirm the results of the eigenvalue computations presented herein. 


\section{Acknowledgments}

The authors are grateful to Dr C.-L. Chang for his assistance and for useful technical discussions. The present work was supported by the Supersonic Cruise Efficiency (Airframe) discipline of the Supersonics Project under NASA's Fundamental Aeronautics Program, with Ms. Linda Bangert as the Associate Principal Investigator for this discipline.

\section{References}

1. Pfenninger, W. and Bacon, J. W., "Ampilfied Laminar Boundary Layer Oscillations and Transition at the Front Attachment Line of a $45^{\circ}$ Flat-nosed wing with and without boundary Layer Suction", Viscous Drag Reduction, ed. C. S. Wells, Plenum, 1969.

2. Mack, L. M. "Boundary Layer Stability Theory", Report 900-277, Rev. A, Jet Propulsion Laboratory, Pasadena, CA, November 1969.

3. Malik, M. R., Li, F. and Chang C.-L., "Crossflow Disturbances in Three-Dimensional Boundary Layers: Nonlinear Development, Wave Interaction and Secondary Instability." J. Fluid Mech. Vol. 268, 1994, pp. 1-36.

4. Bushnell, D.M. and Malik, M.R., "Application of Stability Theory to Laminar Flow Control - Progress and Requirements," Stability of Time Dependent and Spatially Varying Flows," D.L. Dwoyer \& M.Y. Hussaini, eds., Springer Verlag, pp. 1-17, 1987.

5. Malik, M. R., "Hypersonic Flight Transition Data Analysis Using Parabolized Stability Equations with Chemistry Effects" J. of Spacecrfat and Rockets 40,2003, pp. 332-344.

6. Reibert, M. ,Saric , S. Carrillo, R.B., Jr and Chapman, K. L. "Experiments in Nonlinear Saturation of Stationary Crossflow Vortices in a Swept-wing Boundary Layer,” AIAA Paper 96-0184, 1996.

7. Kohama, Y. Ondodera, T. and Egami Y. "Design and Control of Crossflow Instability Field," In Proc. IUTAM Sym. On Nonlinear Instability and Transition in Three Dimensional Boundary Layers," Manchester, U.K (ed. P.W. Duck and P. Hall). Pp- 147-156. Kluwer, 1996

8. Saric, W.S., Reed H.L., and White E.B. "Stability and Transition of Three-Dimensional Boundary Layers," Ann. Rev. Fluid Mech., vol. 35, pp. 413-440, 2003.

9. Saric, W.S., "Gortler Vortices," Ann. Rev. Fluid Mech., vol. 26, pp. 379-409, 1994.

10. Malik, M. R., Li, F. Choudhari, M. M. and Chang C.-L., "Secondary Instability of Crossflow Vortices and Swept-wing Boundary-layer Transition." J. Fluid Mech. Vol. 399, 1999, pp. 85- 115.

11. Choudhari, M., Chang C.-L, Streett C.L., and Balakumar, P., "Integrated Transition Prediction: A Case Study in Supersonic Laminar Flow Control," AIAA Paper 2003-0973, 2003.

12. Choudhari, M, Chang, C.-L., and Jiang, L., "Towards Transition Modeling for Supersonic Laminar Flow Control," Philosophical Transactions of Royal Society of London (Physical and Mathematical Sciences), vol. 363, no. 1830, pp. 1041-1259, 2005.

13. El-Hady, N.M. "Spatial three-dimensional secondary instability of compressible boundary-layer flows," AIAA Journal, vol. 29, no.5, pp. 688-696, 1991.

14. Saric, W. S. and Reed, H. L., "Supersonic laminar flow control on swept wings using distributed roughness." AIAA paper 2002-147, 2002.

15. Hall, P., and Horseman, N., "The Linear Inviscid Secondary Instability of Longitudinal Vortex Structures in Boundary Layers, ” J. Fluid Mech., vol. 232, pp. 357-391, 1991.

16. Chang, C.-L., Malik, M. R. and Vinh, H. "Linear and Nonlinear Instability of Compressible Swept-Wing Boundary Layers.” AIAA Paper 95-2278, 1995.

17. Li, F. and Malik, M.R., "Fundamental and Subharmonic Secondary Instability of Gortler Vortices,", J. Fluid Mech, vol 297, pp.77-100, 1995

18. Lin, R.-S., Malik, M. R., "On the Stability of Attachment-line boundary Layers Part 1. The Incompressibbe Swept Hiemenz Flow." J. Fluid Mech. Vol. 311, 1996, pp. 239-255.

19. Lin, R.-S., Malik, M. R., "Stability and Transition in Compressible Attachment-Line Boundary-Layer Flow", SAE Technical Paper No. 952041, Aerotech '95, Los Angles, CA, September 18-21, 1995.

20. Theofilis, V., "Advances in global linear instability analysis of nonparallel and three-dimensional flows," Progress in Aerospace Sciences, vol. 39, no. 4, pp. 249-315, May 2003.

21. Janke, E. and Balakumar, P., "On the secondary Instability of Three-Dimensional Boundary Layers," Theoret. Comput. Fluid Dynamics, vol. 14, pp167-194, 2000.

22. L. Jiang, M. Choudhari, C.-L. Chang, and C. Liu, "Direct Simulation of Instability-Wave Generation and Propagation in Supersonic Boundary Layers," in Proceedings of International Conference on Computational Science and Its Applications - ICCSA 2003, Montreal, Canada, May 18-21, 2003, Vol. II (Lecture Notes in 
Computer Science 2668), V. Kumar, M.L. Gavrilova, C.J. K. Tan, and P. L'Ecuyer (Eds.), Springer Verlag, Berlin-Heidelberg, July 2003.

23. L. Jiang, M. Choudhari, C.-L. Chang, and C. Liu, "Direct Numerical Simulation of Crossflow Disturbances in Supersonic Boundary Layer," AIAA Paper 2004-0589, 2004.

24. Chang, C.-L., "The Langley Stability and Transition Analysis Codes (LASTRAC: LST, Lineara dn Nonlinear PSE for 2D, Aximsymetric, and Infinite Swept Winga Boundary Layers", AIAA Papert 2003-0974, 2003.

25. Reibert, M. Saric, W. S., Carrilo, R. B., Jr \& Chapman, K. L. 1996 Experiments in Nonlinear Saturation of Stationary Crossflow Vortices a a Swept Wing Boundary Layer. AIAA paper 96-0184.

26. Bonfigli, G, and Kloker, M., "Secondary instability of crossflow vortices: validation of the stability theory by direct numerical simulation," J. Fluid Mech., Vol. 583, pp. 229-272, 2007.

27. Carrillo, R. B., Jr, Reibert, M. S. \& Saric, W. S. 1996 "Distributed Roughness Effects on Stability and Transition in Swept Wing Boundary Layers," Final report for NASA LaRC co-op. agreement NCC-1-194.

28. White E. B., and Saric W. S., "Secondary Instabilty of Crossflow Vortices." J. Fluid Mech. Vol. 525, pp. 275308, 2005.

29. Chang, C.-L., and Choudhari, M, "Boundary-Layer Receptivity and Integrated Transition Prediction," AIAA Paper 2005-0526, 2005.

30. Theofilis, V., Fedorov, A.V. and Collis, S. S., "Leading-Edge Boundary Layer Flow, Prandtl's Vision, Current Developments and Future Persepectives," International Union of Theoretical and Applied Mechanics Symposium: One Hundred Years of Boundary Layer Theory, August 2004.

31. Powell, A., Beeler, G., and King, R.A., "Attachment-Line Tripping Due to Suction Through a Micro-Perforated Skin at Mach 2," AIAA Paper 2006-3221, 2006.

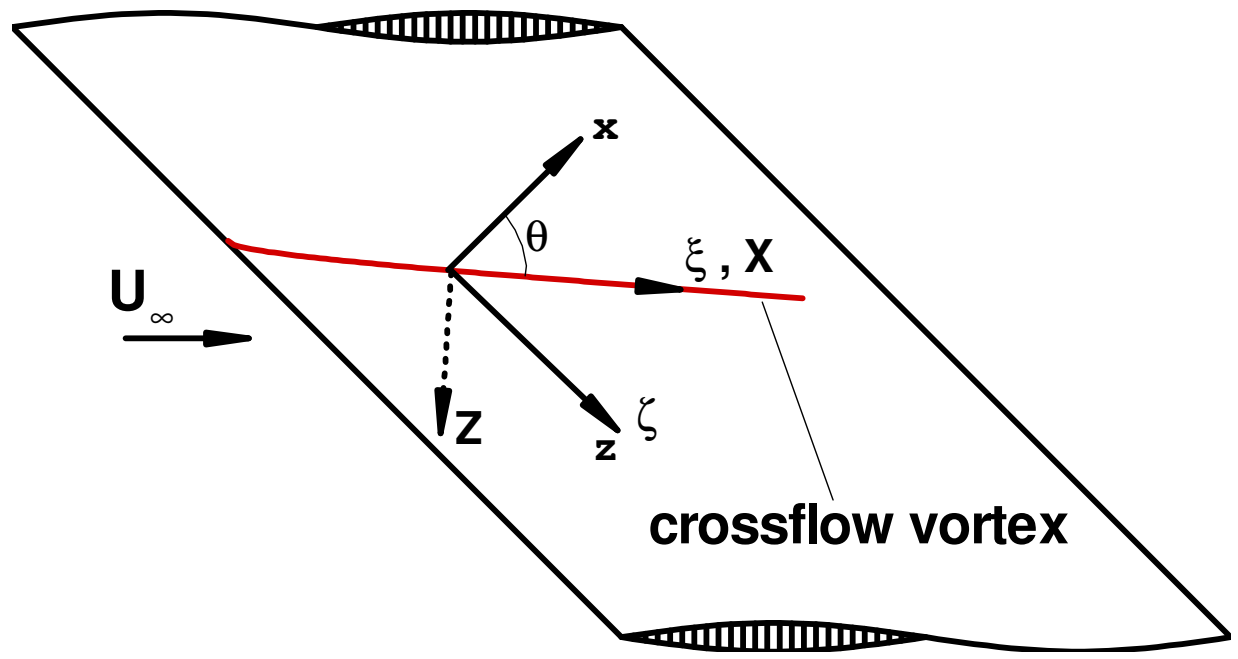

Figure 2.1. Schematic of a swept wing of infinite span, with three surface coordinate systems, namely, $(x, z),(X, Z)$ and $(\xi, \zeta)$, the last of which corresponds to the non-orthogonal, vortex-aligned coordinate system. 


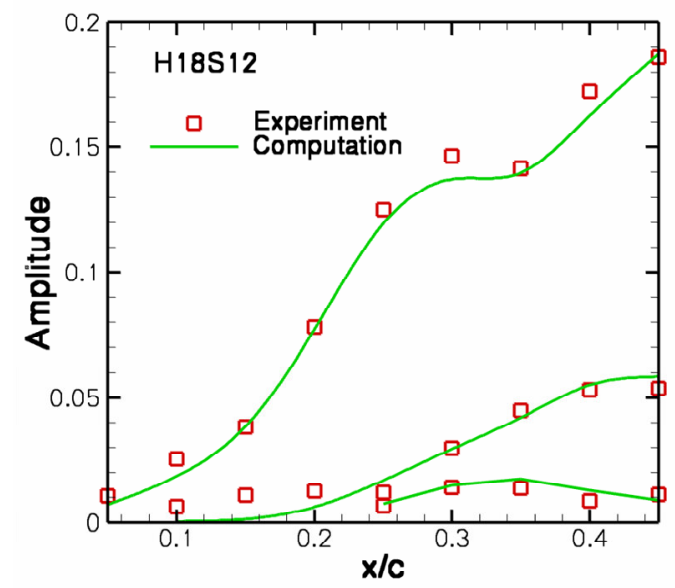

Figure 3.1 (a) Comparison of experimental and computational modal amplitudes for $12 \mathrm{~mm}$ crossflow vortices in case H18S12.
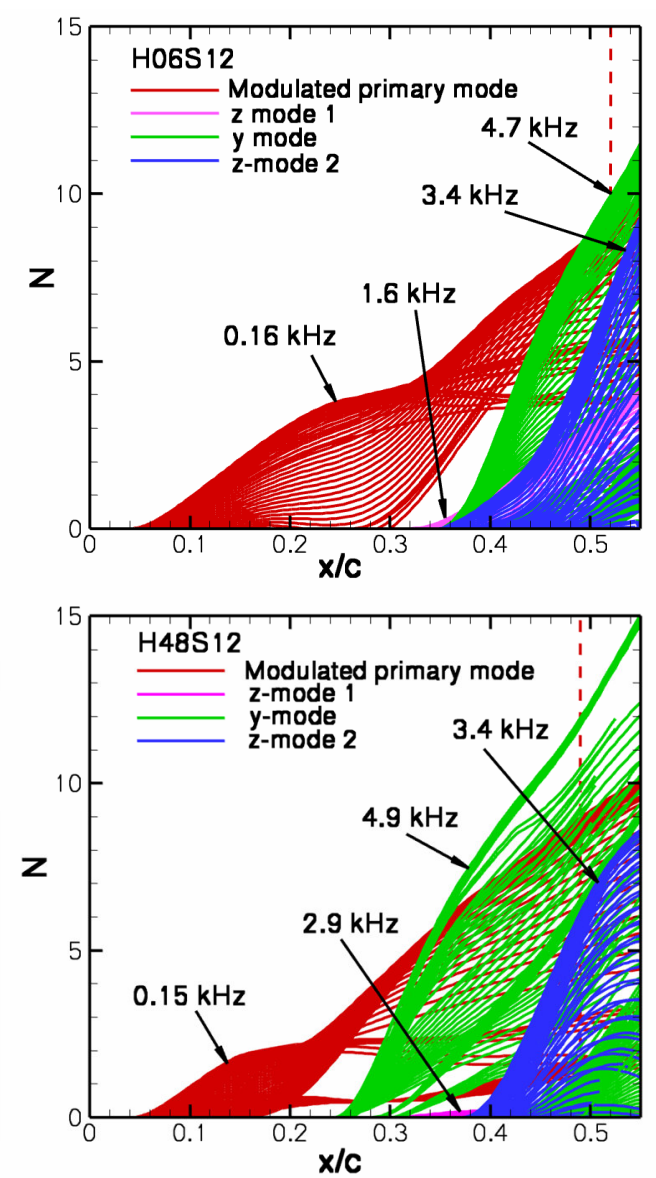

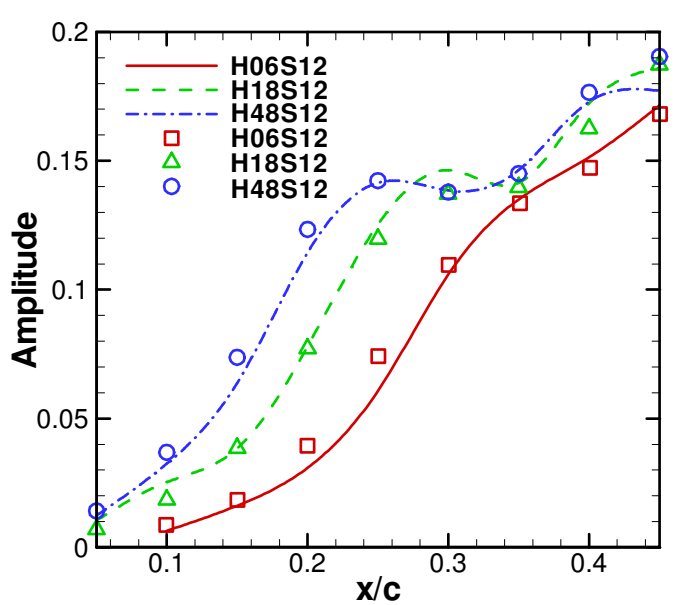

Figure 3.1 (b) Comparison of measured and predicted fundamental amplitudes for cases H06S12, H18S12, and H48S12 (curves: computation, symbols: experiment).
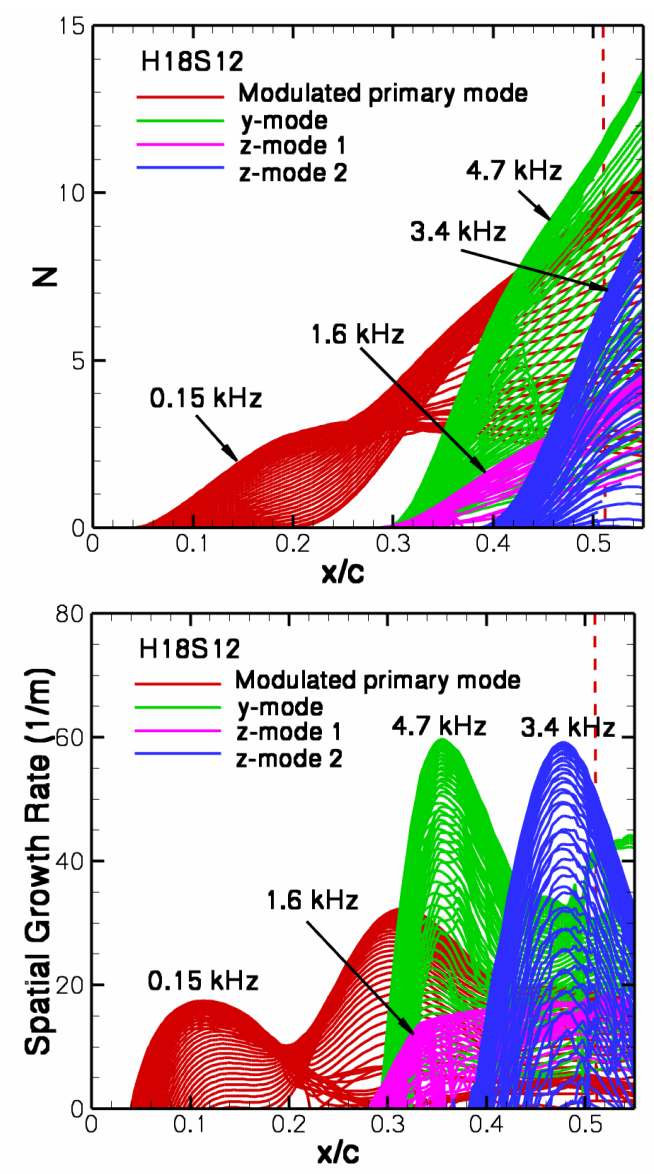

Figure 3.2 Top left, top right and bottom left: $\mathrm{N}$-factor curves for selected modes for 6-, 18- and 48-micron roughness cases, respectively. Bottom right: spatial growths rates for the 18-micron case. Measured transition locations are indicated by a dashed red line. Color of $\mathrm{N}$-factor curves denotes the mode type, whereas different curves for a given mode correspond to disturbances at different frequencies. The most amplified frequency for each selected mode is highlighted on the plot. 


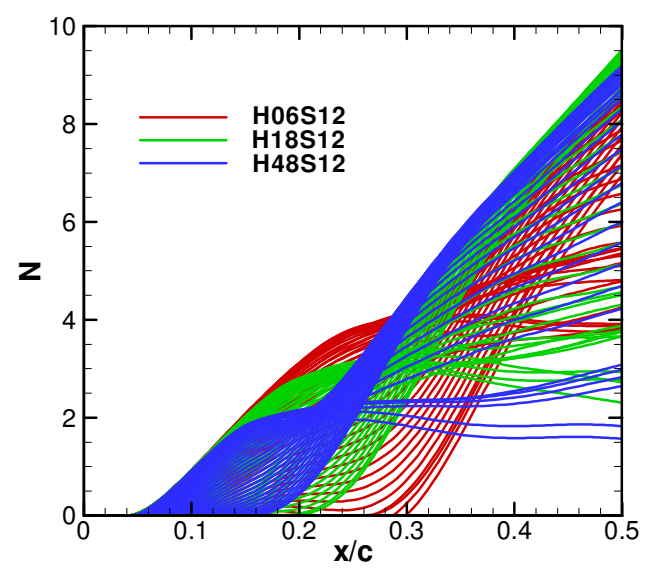

Figure 3.3 (a) $N$-factors for modulated traveling crossflow vortices for different roughess heights. Color of curve indcates roughness height, whereas different curves of the same color are for different frequencies.

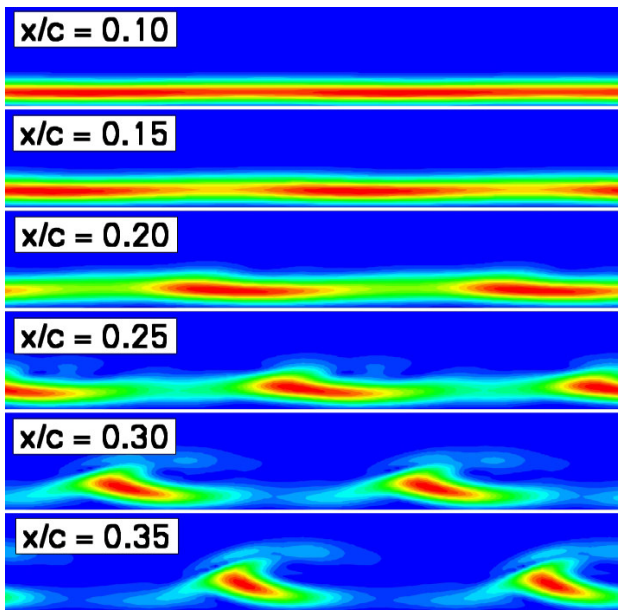

Figure 3.4 Eigenfunction evolution for modulated traveling mode at $f=200 \mathrm{~Hz}$. The abscissa and ordinate correspond to suitably normalized spanwise coordinate $\zeta$ and wall-normal coordinate y, respectively.

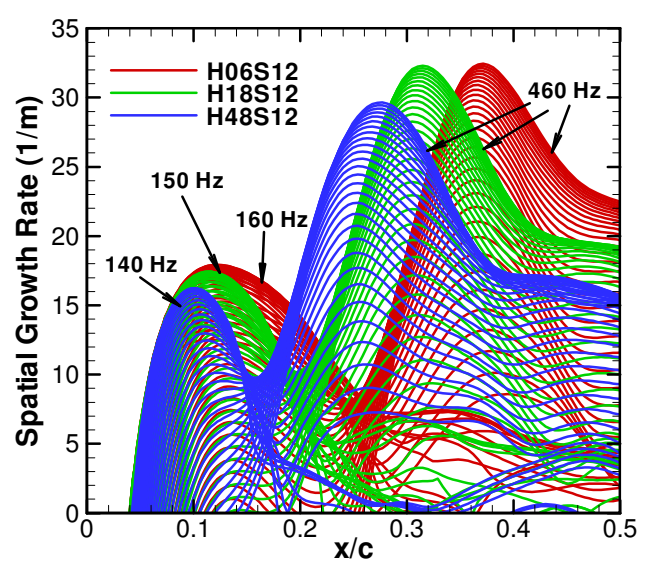

Figure 3.3 (b) Growth rates of modulated traveling crossflow vortices corresponding to cases in Fig. 3.3(a). Peak growth-rate frequency in each case is indicated.

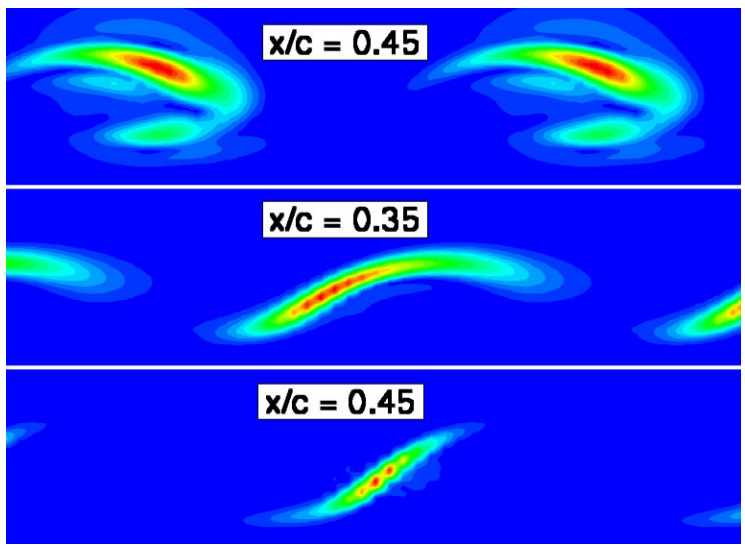

Figure 3.5 Magnitude of secondary instability eigenfunctions for velocity component along the vortex axis for 45-deg swept NLF-0415(b) wing. Top: y-mode, middle: z-mode 1 and bottom: z-mode 2 (Abscissa and ordinate similar to Fig. 3.4).
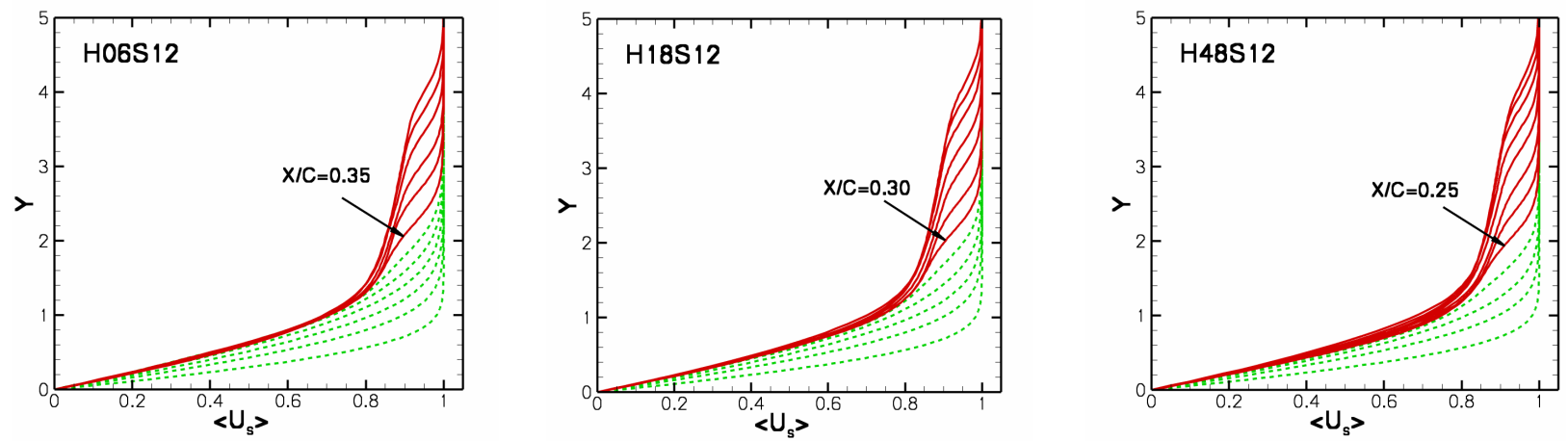

Figure 3.6. Mean velocity profiles aligned with the local inviscid streamline. Profiles begin at $x / c=0.05$ and end at $x / c=0.6$, with an increment of 0.05 across successive profiles. Highlighted profile locations indicate where the profile develops a distinctly inflectional character. 


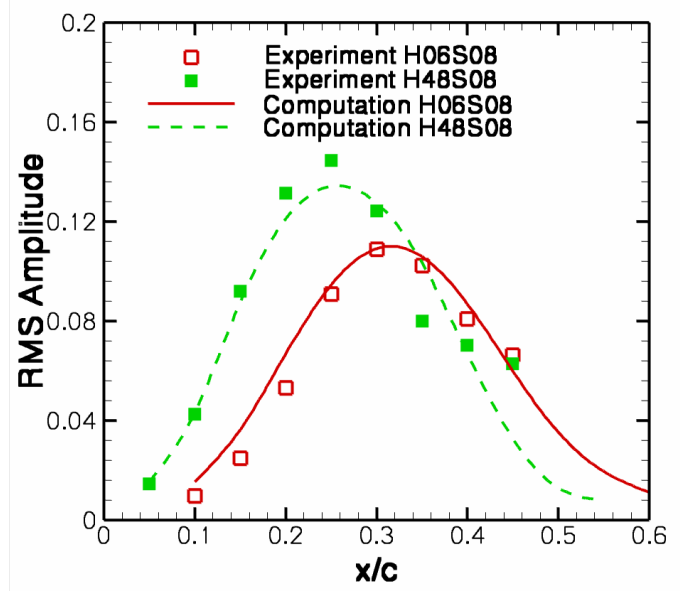

Figure 3.7. Comparison of experimental and computational r.m.s. amplitudes of stationary disturbance for a roughness array spacing of $\lambda_{2}=8 \mathrm{~mm}$ (cases H06SO8 and H48SO8 for the NLF0415(b) configuration).

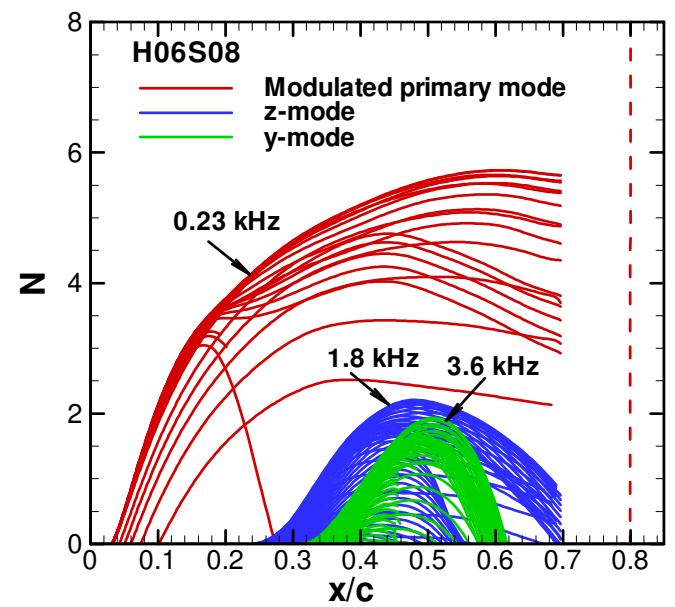

(a) 6-micron roughness

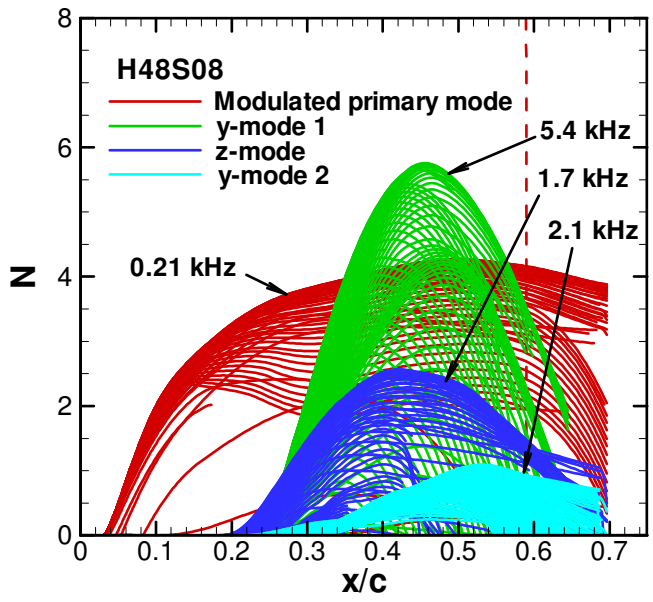

(b) 48-micron roughness.

Figure 3.8. $N$-factor curves for $\lambda_{2}=8 \mathrm{~mm}$ (dashed line indicates measured transition location; line color denotes selected mode type, whereas different curves of same color indicate different frequencies for a given mode; highlighted frequencies correspond, approximately, to most amplified frequency for each selected mode). 


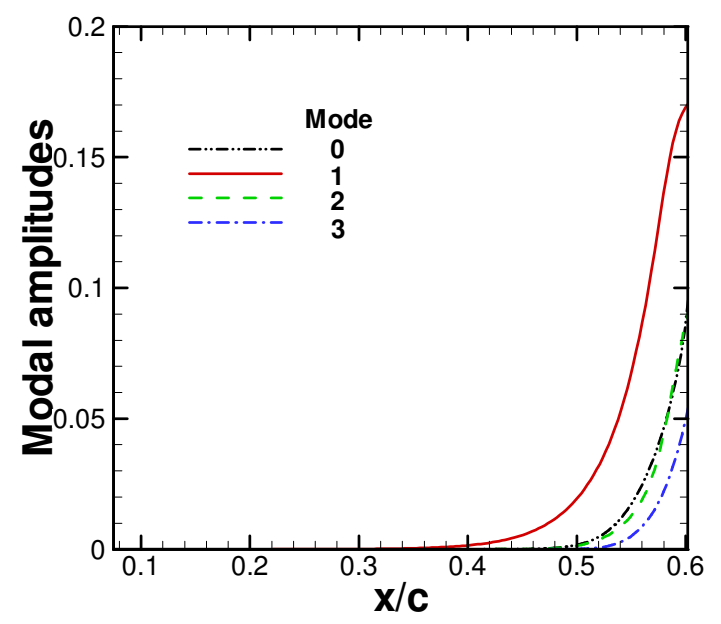

(a) $\lambda_{z}=3 \mathrm{~mm}$

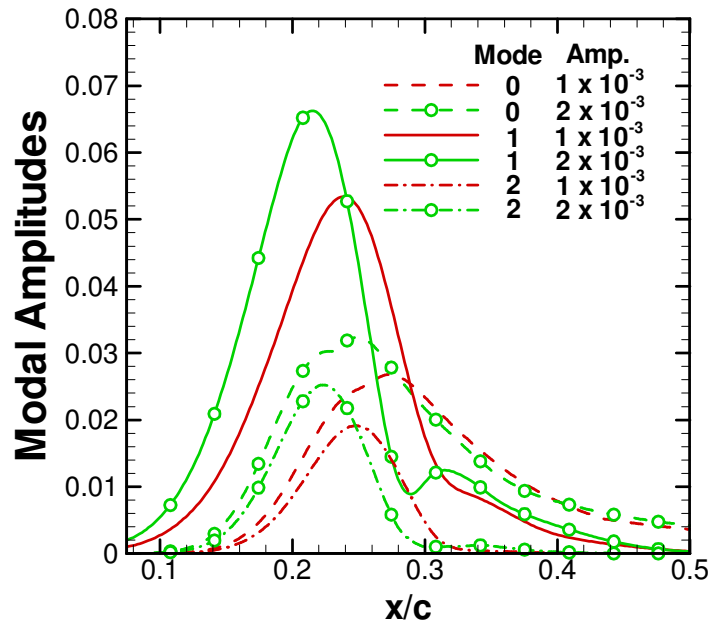

(b) $\lambda_{z}=1.5 \mathrm{~mm}$ for initial fundamental amplitudes of 0.001 and 0.002

Figure 4.1. Primary disturbance evolution along Mach 2.4 swept-wing boundary layer. Amplitudes of several disturbance harmonics, including the fundamental mode (mode 1) and mean-flow-correction (mode 0), are shown in each figure.

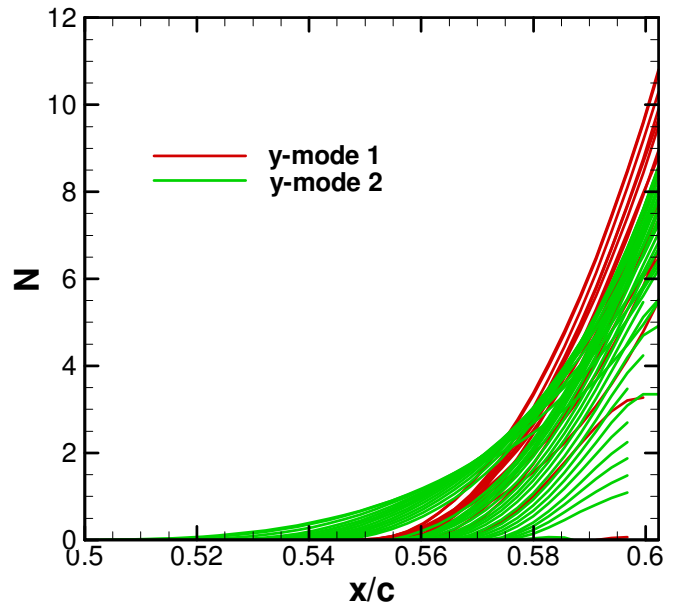

Figure 4.2(a) $\mathrm{N}$-factor curves.

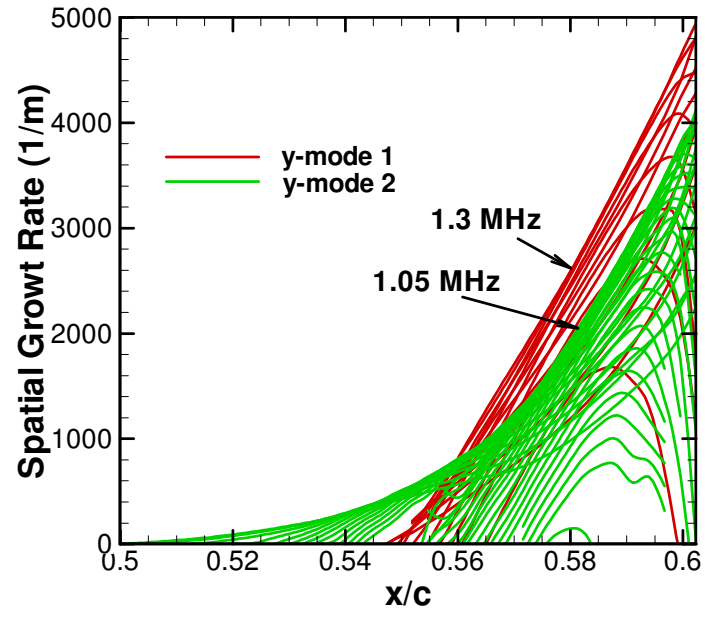

Figure 4.2(b) Spatial growth rate curves.

Figure 4.2. Secondary instability of stationary crossflow mode at $\lambda_{z}=3 \mathrm{~mm}\left(A_{i}=1 e-7\right.$ in Fig. 4.1(a)). Line color denotes selected mode type, whereas different curves of same color indicate different frequencies for that mode; highlighted frequencies correspond, approximately, to most amplified frequency for each selected mode. 


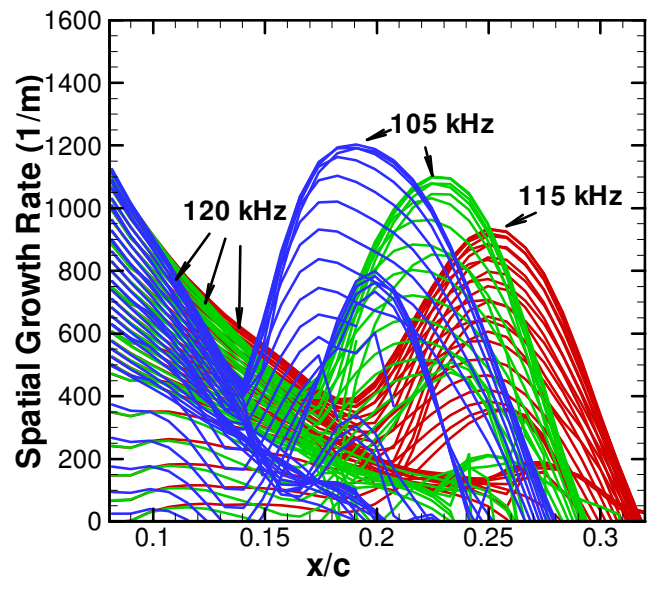

Figure 4.3. Effect of initial amplitude of the stationary crossflow vortex on growth rates of modulated traveling crossflow instability at $\lambda_{z}=1.5 \mathrm{~mm}$ (Line color denotes initial amplitude. Red curve: $A_{i}=0.001$, Green: $A_{i}=$ 0.002, and Blue: $A_{i}=0.005$. Different curves at each initial amplitude represent different frequencies, with peak growth rate frequencies indicated on the plot).

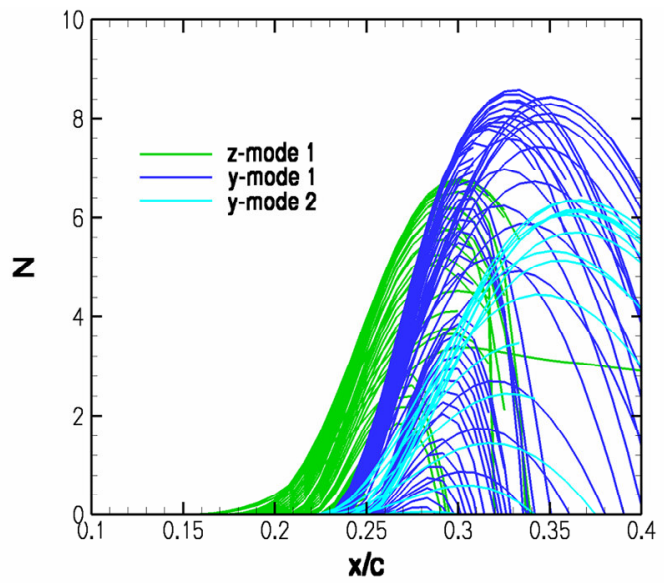

Figure 4.4(a) $N$-factors curves, $A_{i}=0.001$

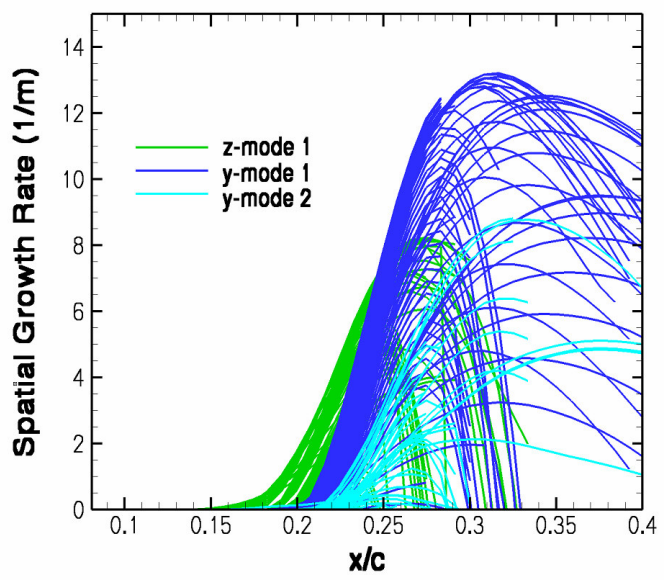

Figure 4.4(c) $N$-factors curves, $A_{i}=0.002$

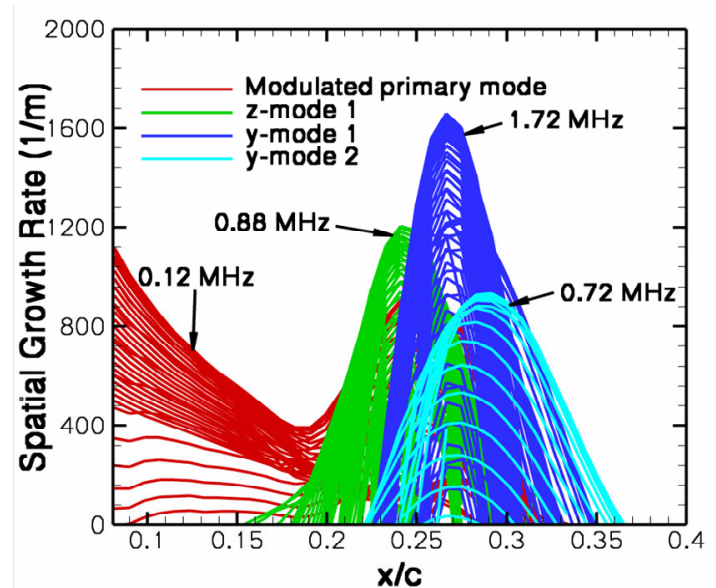

Figure 4.4(b) Spatial growth rate curve, $A_{i}=0.001$

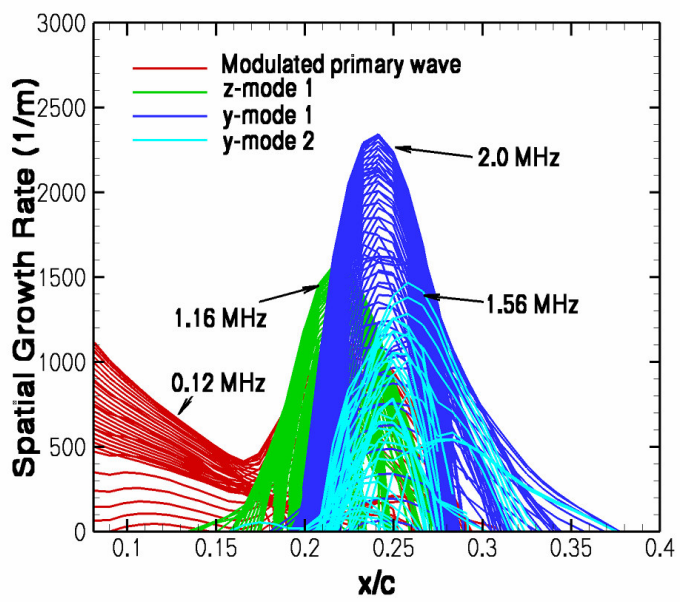

Figure 4.4(d) Spatial growth rate curves, $A_{i}=0.002$.

Figure 4.4. Secondary instability of stationary crossflow mode at $\lambda_{z}=1.5 \mathrm{~mm}$. (Different curves of the same color are for different frequencies, and peak growth rate frequencies for each mode are indicated in parts $b$ and d). 

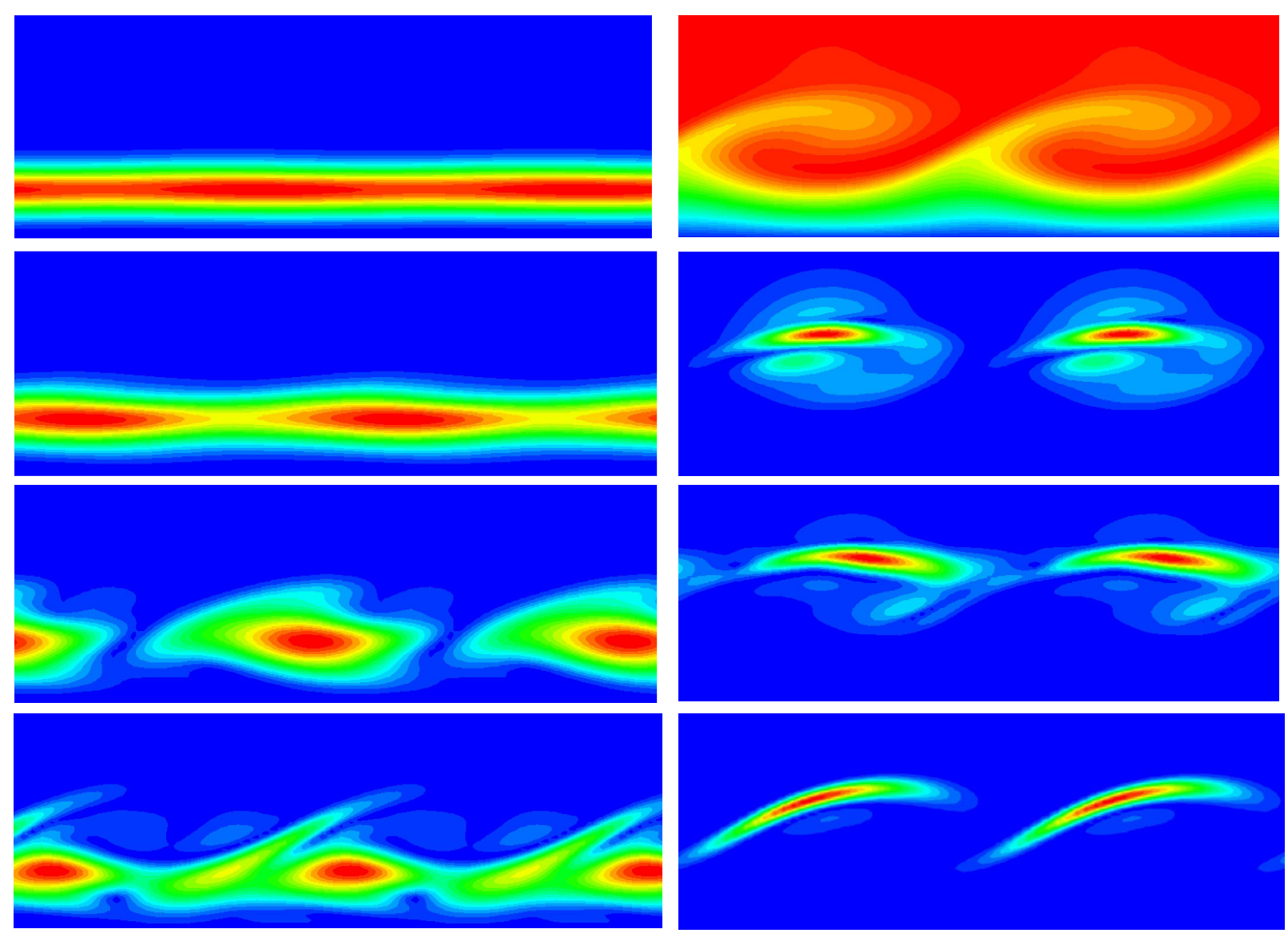

Figure 4.5. Eigenfunction contours for modulated traveling crossflow waves at $120 \mathrm{kHz},\left(\lambda_{z}=1.5 \mathrm{~mm}\right.$; $x / c \approx 0.08,0.11,0.16$, and 0.25 from top to bottom, respectively). Abscissa and ordinate similar to Fig. 3.4; Contour variable corresponds to magnitude of eigenfunction for the velocity component along the direction of stationary vortex (which is approximately aligned with the local inviscid streamline).

Figure 4.6. Basic state and secondary mode shapes at $\mathbf{x} / \mathbf{c}=0.25\left(\lambda_{z}=1.5 \mathrm{~mm}\right) . \quad$ Abscissa and ordinate similar to Fig. 3.4. Top: Contours of velocity component along the axis of the stationary crossflow vortex; Upper and lower middle, and bottom: Secondary instability eigenfunction corresponding to velocity component along the vortex axis for two $y$ modes and one z-mode, respectively, at $f=1700 \mathrm{kHz}$.
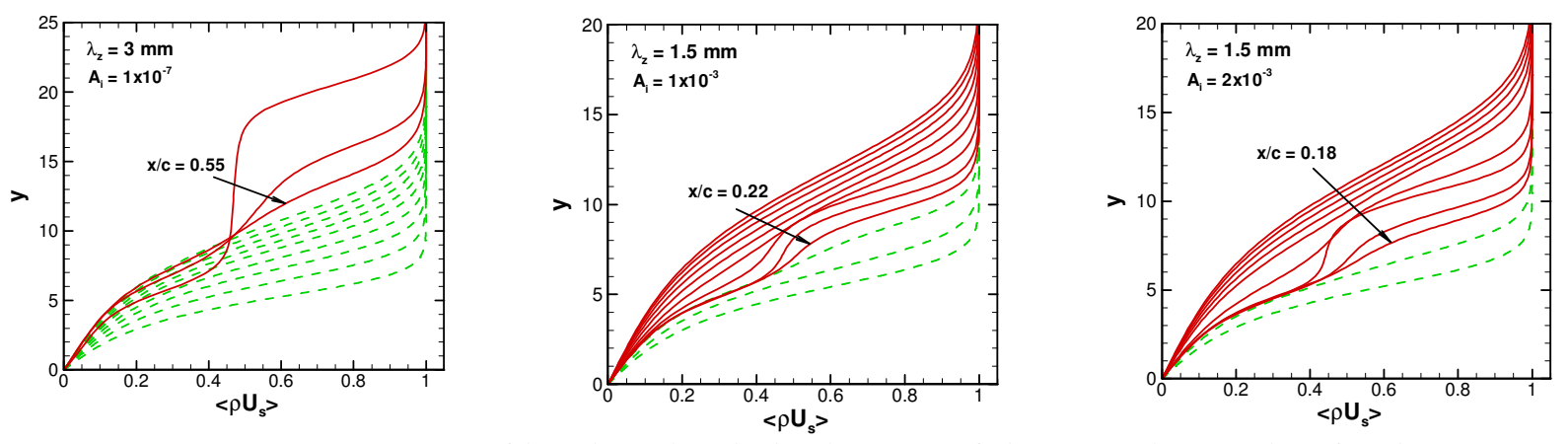

Figure 4.7 Mean momentum profiles aligned with the direction of the inviscid streamline for the supersonic swept-airfoil configuration $\left(\lambda_{z}=1.5 \mathrm{~mm}\right)$, with highlighted locations indicating where the profile first develops significant inflection. Profiles start at $x / c=0.1$ and proceed in intervals of $\Delta x / c=0.05$, with the exception of $x / c=0.22$ (middle plot) and $x / c=0.18$ (right plot). 


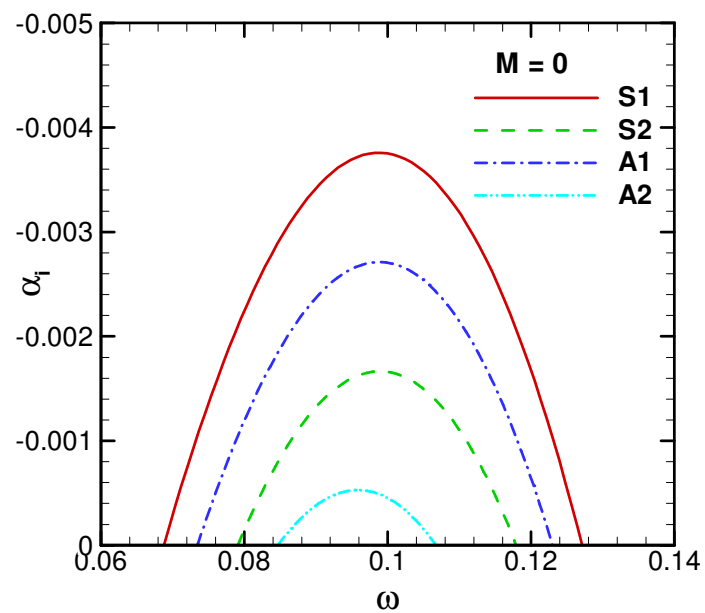

Figure 5.1. Spatial growth rates for modes S1, A1, S2 and A2 of attachment line instability for swept Hiemenz flow. $M=0, \operatorname{Re}=800$.

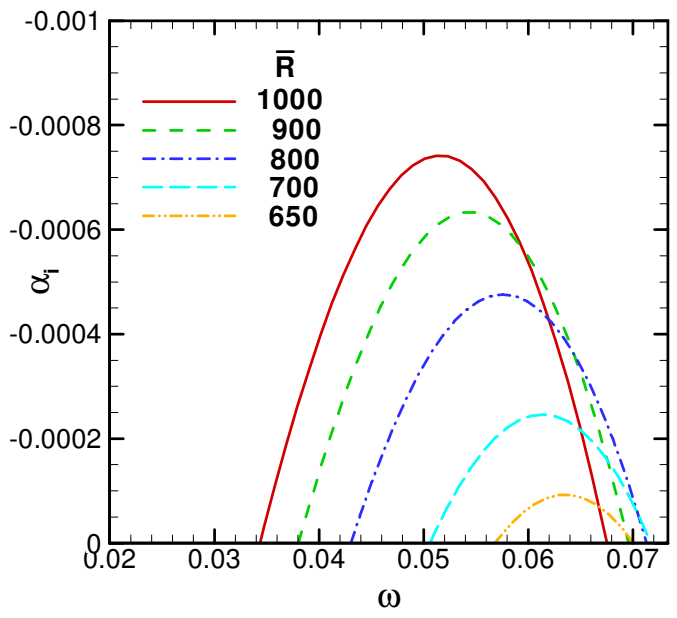

(a) Spatial growth rate

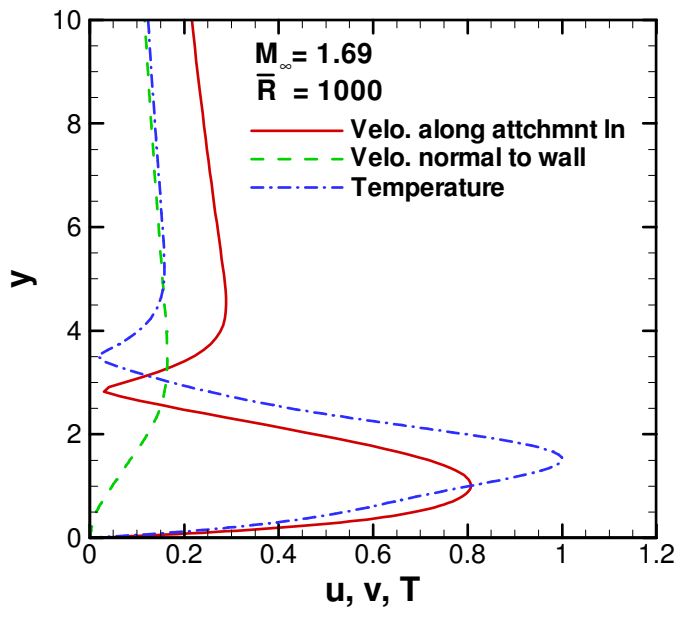

(b) Mode shapes at attachment line

Figure 5.2. Spatial instability characteristics of mode S1 of attachment line instability for supersonic swept Hiemenz flow. $M_{e}=1.69$, adiabatic wall. 